Proyecciones

Vol. 26, $\mathrm{N}^{o}$ 1, pp. 105-142, May 2007.

Universidad Católica del Norte

Antofagasta - Chile

\title{
MAXWELL REVISITED
}

\author{
NORBERTO SÁINZ \\ Pontificia Universidad Católica de Valparaíso, Chile.
}

Received: December 2006. Accepted : March 2007

\begin{abstract}
This work explores what other mathematical possibilities were available to Maxwell for formulating his electromagnetic field model, by characterizing the family of mathematical models induced by the analytical equations describing electromagnetic phenomena prevailing at that time.

The need for this research stems from the article "Inertial Relativity - A Functional Analysis Review", recently published in "Proyecciones", which claims and demonstrates the existence of an axiomatic conflict between the special and general theories of relativity on one side, and functional analysis on the other, making the reformulation of the relativistic theories, mandatory. As will be shown herein, such reformulation calls for a revision of Maxwell's electromagnetic field model.

The conclusion is reached that -given the set of equations considered by Maxwell- not a unique, but an infinite number of mathematically correct reformulations to Ampère's law exists, resulting in an equally abundant number of potential models for the electromagnetic phenomena (including Maxwell's). Further experimentation is required in order to determine which is the physically correct model.
\end{abstract}




\section{INTRODUCTION AND PRESENTATION OUTLINE}

\subsection{Introduction}

The article "Inertial Relativity - A Functional Analysis Review" [1], recently published in "Proyecciones", claims and demonstrates the existence of an axiomatic conflict between the special and general theories of relativity (STR and GTR) on one side, and functional analysis on the other. Due to the fundamental nature of this conflict -the cited article concludesin order to perform legitimate analytical operations in spacetime, its traditional relativistic topologies must be abandoned and both theories, the STR and GTR, must be reformulated.

It is well-known, however, that there was no room for options in the formulation of the relativistic theories. Einstein was inexorably led -first into the STR, then into the GTR- by his insightful and univocal combination of the then-prevailing-model for the propagation of light, in conjunction with two well-established physical principles:

- The principle of relativity ${ }^{1}$.

- The principle of universal covariance ${ }^{2}$.

The implication of such univocity is that, for as long as functional analysis stands valid, either an electromagnetic model alternative to Maxwell's exists, or at least one of the physical principles supporting Einstein's work must be dropped.

But dropping any of the well-established physical principles would lead to extreme consequences of epistemological and philosophical nature, not to mention the implications on Einstein's remarkable contribution. So the least traumatic way to proceed is to look for alternatives to the thenprevailing (and still current) electromagnetic field model formulated in 1864 - 1865 by Maxwell [5].

One such alternative was proposed by Hertz in his paper, "Über die Grundgleichungen der Elektrodynamik für bewegte Körper" published in 1890 in Annalen der Physik, [6]. This model is based in postulating that

\footnotetext{
${ }^{1}$ In [2] Einstein makes use of the principle of relativity to establish that Lorentzian transformations must apply between inertial reference frames and in [3] he demonstrates the Maxwell equations' covariance upon a Lorentzian reference frame transformation.

${ }^{2}$ In [4] Einstein uses the principle of equivalence (an extension of the principle of relativity to accelerated frames) in conjunction with what he calls the principle of universal covariance upon a (Lorentzian) frame transformation to establish the applicable metric.
} 
Faraday's and Ampère's relations be expressed in terms of total (instead of partial) time derivatives by means of the incorporation of three velocitytype parameters, which Hertz named $\alpha, \beta, \gamma$ and associated to the velocity coordinates of the later questioned æther, relative to the laboratory frame. Though not proved in his paper, under certain modern assumptions respect of the physical meaning of the velocity-type parameters, Hertz' formulation may be considered invariant before Galilean reference frame transformations and therefore, from the point of view of the conflict herein addressed, may be argued to provide with one working alternative. However, because Hertz' model is well-known, and its merits and demerits have been thoroughly considered and debated, this work does not pursue further along this line of thought.

Instead, this paper contributes by exploring what other mathematical possibilities were available to Maxwell when formulating his well-known electromagnetic field model, which resulted in a modification to Ampère's $l_{a w}{ }^{3}$. It does so by characterizing the family of mathematical models induced by the analytical equations describing electromagnetic phenomena prevailing at that time. The conclusion is reached, that a family of mathematically correct reformulations to Ampère's law exists, resulting in an infinite number of candidate (or potential) models for the propagation of light, including Maxwell's electromagnetic field model. Further experimentation is required in order to determine which is the physically correct model.

Because Maxwell detected no alternatives in his work, the question of identifying the physically correct model out of the family of mathemati-

\footnotetext{
${ }^{3}$ It can hardly be said that Maxwell modified Ampère's law when in fact he never considered it during the development of his electromagnetic field model. If he mentioned once Ampère's name and contribution during said process in his 1865 paper [7], it was only to say that:

"If, therefore, the phenomena described by Faraday in the Ninth Series of his Experimental Researches were the only known facts about electric currents, the laws of Ampère relating to the attraction of conductors carrying currents, as well as those of Faraday about the mutual induction of currents, might be deduced by mechanical reasoning."

Notwithstanding, because one important consequence of Maxwell's work was the addition of the displacement current to the Equation of Electric Currents -which derives directly from Ampère's law and today carries Ampère's name as a member of the Maxwell equations- and because such addition is by far better discussed in Maxwell's 1873 Treatise [8] than in his 1865 paper (though some may argue that this is a matter of taste), the Treatise and its line of thought will be used in this work, declaring for simplicity that Maxwell modified Ampère's equation.
} 
cally correct candidates was never posed, so ways to an answer were never proposed, and therefore the missing experiment is long overdue.

\subsection{Presentation Outline}

The family of mathematical models induced by the analytical equations describing electromagnetic phenomena prevailing at the time of Maxwell is herein characterized by means of a "gedanken", or "thought" -experiment, detailed in $\S 2$.

Thought-experiments depend on the existence of some well-established model that enables describing, in a consensually accepted manner, the way in which the thought-experiment should unfold. In this manner, if proper logical and mathematical operations are applied on the accepted model, acceptable conclusions result.

Since the prevailing electromagnetic field model is herein under scrutiny, it cannot be used as the required, well-established model. The research must delve back into such foundations of electricity and magnetism that generate no questions regarding their applicability, coherence and mathematical correctness. Considering that circa 1865, competing opinions and formulations existed for fundamental concepts and expressions of the emergent electromagnetic theory ${ }^{4}$, and given the fact that this work aims to contribute with a mathematical (as opposed to a physical) review of Maxwell's conclusions, the concepts and equations selected by Maxwell as the fundaments of his theory (or mathematically coherent with Maxwell's model) have been selected as a starting point.

Modern terminology is used when referring to said concepts, and modern notation is used for writing said equations, even though the $19^{\text {th }}$ century physicists whose work is herein considered never employed either of them. In a similar manner, the differential forms of the mathematical expressions are herein used, due consideration given to the work of Heaviside, Lorentz and others, who formulated what is now called microscopic electro-

\footnotetext{
${ }^{4}$ While Ampère [9] believed that the element of force between two sections of currentcarrying circuit was directed along their connecting radius, Neumann [10], [11] and Weber [12], [13] led to competing differential expressions (which rendered equivalent results upon integration along closed circuits) for the elemental force and other concepts (such as Faraday's induction and energy between elements of current).

In 1870 Helmholtz [14] demonstrated that Weber's model, while allowing the deduction of correct forces between closed circuits, led to predict non-physical behavior of charges in motion and today, Neumann's expression for the elemental force is most widely used. Weber is now recognized amongst other contributions, for his focus on charged particles instead of fluids to model electric currents.
} 
magnetism and showed its equivalence with Maxwell's macroscopic theory, enabling the alternative formulation and usage of either. The combined name (i.e., Maxwell-Faraday, Maxwell-Gauss) will be used in $\S 3$ and $\S 4$ when referring to these modern terminology differential forms, in order to distinguish them from -honoring at the same time- their authors' original contributions.

In this manner, nine physical relations - detalied in $\$ 1.3$ - have been selected as the cornerstones of this analysis. The first three describe the electromagnetic behavior of materials; the second three describe the relation between time-varying electromagnetic fields and charge distributions, while the last three apply to static fields, currents and charge distributions.

Conjectures will be raised respect the extension of the last three static relations (under 7, 8 and 9 of $\S 1.3$ ) to time-varying fields, and the stated logic (that valid logical and mathematical operations, applied to wellestablished models, yield acceptable results) will be used to confirm or reject such conjectures.

In order to make a full characterization of the fields generated in the thought-experiment - which are functions of time and space- their divergence and curl will be calculated, showing that the analytical expressions for the effects of these operators on the electromagnetic fields may be defined only up to a set of parameters, herein labeled $\lambda_{1}$ and $\lambda_{2}$. Maxwell implicitly assumed a specific value for these parameters: $\lambda_{1}=\lambda_{2}=1$, leading way to his well-known equations. Vector calculus concepts introduced by Heaviside years later show, notwithstanding, that other values of $\lambda_{1}$ and $\lambda_{2}$ may be applicable ${ }^{5}$.

As a result, the following conclusions are obtained:

1. Maxwell was right in detecting that the equations describing the electromagnetic phenomena prevailing at that time were mathematically inconsistent and required a revision.

2. Maxwell's proposition, which resulted in a modification of Ampère's original equation for the case of time-varying fields, was incomplete. A complete, mathematically consistent revision of the prevailing set of equations describing the electromagnetic phenomena includes two scalar parameters.

\footnotetext{
${ }^{5}$ It was not evident at the time Maxwell formulated his electromagnetic field equations, that -except for a trivial term- continuously differentiable $3-\mathrm{D}$ vector fields are well-defined by their divergence and curl. The discovery of this feature of continuously differentiable 3-D fields (owed to the work of Heaviside and others) will prove to contribute -with substantial knowledge- to this ex-post analysis.
} 
3. These two scalar parameters generate a family of candidate electromagnetic field models, all the members of which are mathematically consistent with the originating set of assumptions and therefore, from a mathematical point of view, undistinguishable.

4. Not all the members of the above family are physically adequate electromagnetic field models, as neither are all values of the scalar parameters. The physically correct models, jointly with the corresponding set of proper parameter values, are to be experimentally determined.

\subsection{Assumptions}

1. Ohm's equation of resistance is assumed valid in its differential form for time-invariant and time-varying fields ${ }^{6}$.

2. The equation of electric permeability is assumed valid in its differential form for time-invariant and time-varying fields ${ }^{7}$.

3. The equation of electric elasticity (the concept evolved into today's permittivity) is assumed valid in its differential form for time-invariant and time-varying fields ${ }^{8}$.

4. The equation of continuity for electric charges in its differential form is assumed valid for time-invariant and time-varying fields ${ }^{9}$.

5. Gauss' equation in its differential form is assumed valid for timeinvariant and time-varying fields ${ }^{10}$.

6. Faraday's equation in its differential form is assumed valid for timeinvariant and time-varying fields ${ }^{11}$.

7. Ampère's original equation in its differential form is assumed valid for time-invariant fields ${ }^{12}$.

8. Biot-Savart's equation is assumed valid for time-invariant fields ${ }^{13}$.

\footnotetext{
${ }^{6}$ Gave rise to Eqn. (F) in [5], Eqn. (G) in [8].

${ }^{7}$ Defined in Par. (60), gave rise to Eqn. (B) in [5], Eqn. (L) in [8].

${ }^{8}$ Gave rise to Eqn. (E) in [5], Eqn. (F) in [8].

${ }^{9}$ Gave rise to Eqn. (H) in [5].

${ }^{10}$ Gave rise to Eqn. (G) in [5], Eqn. (J) in [8].

${ }^{11}$ Gave rise, jointly with Eqn. (B), to Eqn. (D) in [5], Eqn. (B) in [8].

${ }^{12}$ Coherent with prevailing model for time-invariant fields.

${ }^{13}$ Coherent with prevailing model for time-invariant fields. Evolves into Ampère's law.
} 
9. Coulomb's equation is assumed valid for time-invariant fields ${ }^{14}$.

Conjectures will be raised respect the extension of the assumptions under 7,8 and 9 above to time-varying fields.

${ }^{14}$ Coherent with prevailing model for time-invariant fields. Evolves into Gauss' law. 


\section{THE EXPERIMENT}

\subsection{General description and setup}

This is an elementary thought-experiment, consisting of two stages:

- In the first stage of the experiment, a conducting wire circuit will be submitted to an external time-varying magnetic field.

- In the second stage, the same driving magnetic field will be used, but the wire will be cut and a segment of it will be withdrawn.

The fields generated in both stages of the experiment will be modeled, based on the assumptions of $\S 1.3$.

\subsubsection{First stage}

Consider a wire loop through which an externally generated magnetic field of time-varying intensity, $\mathbf{B}(t, \mathbf{x})$ is applied. As usual, $t$ and $\mathbf{x}$ refer to the temporal and spatial coordinates of an event in the traditional $1+3 D$ coordinate system, fixed in the observer's reference frame.

This external magnetic field induces an electric field in its surroundings, which integrates along the conducting circuit to an emf, driving a current $i(t)$ around the wire, as shown in figure 1.

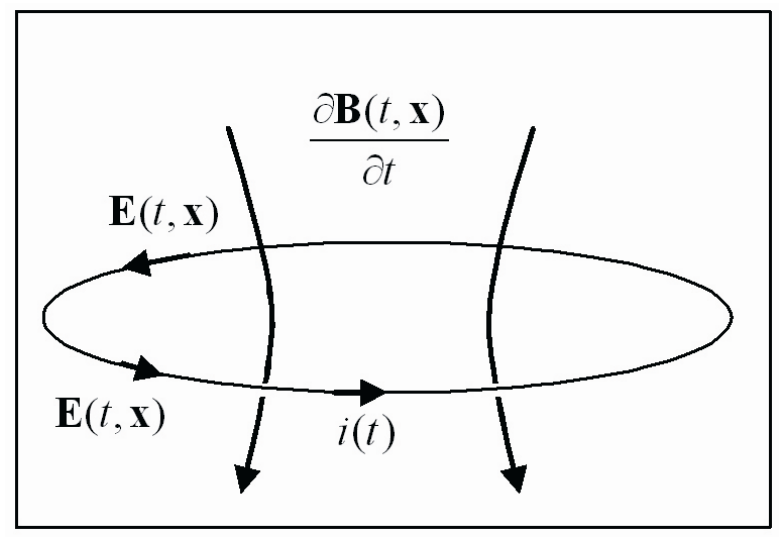

Figure 1 : Magnetic induction in closed loop

The field $\mathbf{E}(t, \mathbf{x})$ represented in figure 1 is considered to exist not only where the conductor is present, but also in the void surroundings wherever the field $\mathbf{B}(t, \mathbf{x})$ varies in time. 


\subsubsection{Second stage}

In the second stage, a piece of the wire is cut, interrupting the circuit. This forces electrical charges to concentrate at the wire ends, as in figure 2.

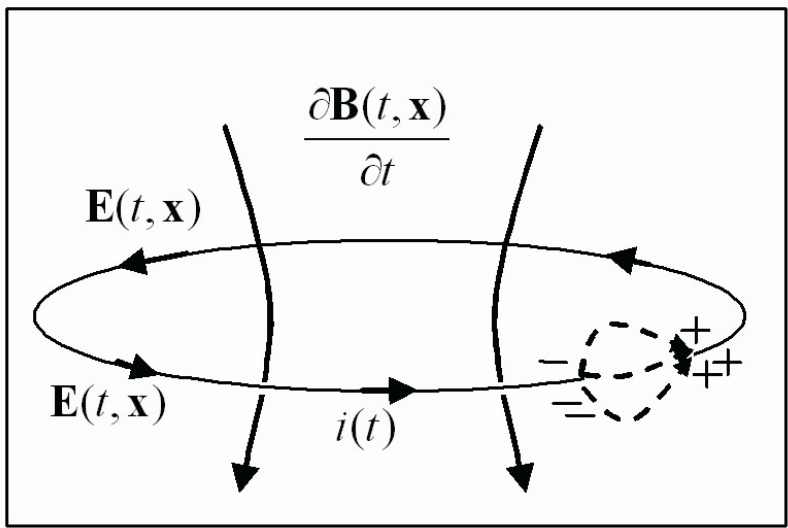

Figure 2 : Magnetic induction in open loop

Before the wire was cut, the electric field was exclusively inductive. After the wire is cut, the electric field close to the wire ends changes, as in figure 2, due to the charge buildup at the discontinuity points. Within the conductor, these charges generate an electric field that opposes and tends to compensate the electric field induced by the external magnetic field. Externally to the conductor, the field generated by the charges tends to contribute to the electric field induced by the magnetic field. The field generated by the charges is conservative, as opposed to the non-conservative field generated by magnetic induction. 


\section{ANALYSIS OF THE RESULTS}

Assumptions numbers 1, 2 and 3 of $\S 1.3$ allow declaring that this analysis is performed for a wire of conductivity $\sigma$ immersed in a medium of permeability $\mu_{0}$ and permittivity $\varepsilon_{0}$. These electromagnetic properties of materials and media are assumed homogeneous and isotropic. The mks physical unit system is used.

\subsection{The Electric Field}

\subsubsection{First stage of the experiment.}

Because Maxwell-Gauss' and Maxwell-Faraday's equations hold (they are assumed valid in 5 and 6 of $\S 1.3$, respectively), if the local electric and magnetic fields were to be measured, any observer could readily verify that the electric field induced in the first part of this experiment is non-conservative and, within the agreed accuracy, satisfies both: the Maxwell-Faraday induction equation,

$$
\nabla \times \mathbf{E}^{T O T}(t, \mathbf{x})=-\frac{\partial \mathbf{B}^{T O T}(t, \mathbf{x})}{\partial t}
$$

and a null Maxwell-Gauss condition,

$$
\nabla \cdot \mathbf{E}^{T O T}(t, \mathbf{x})=0
$$

The latter results may be explained by the electrically neutral conductor used in the experiment. The lack of isolated electrical particles precludes all attempts to enclose unbalanced electric charges in neighborhoods of any relevant ${ }^{15}$ size. Hence, Maxwell-Gauss' law which states:

$$
\nabla \cdot \mathbf{E}^{T O T}(t, \mathbf{x})=\frac{\rho(t, \mathbf{x})}{\varepsilon_{0}}
$$

is reduced to (3.2). Furthermore, since there are no isolated electrical charges, there is no field of a conservative type, i.e., no field generated by electrical charge distributions.

\footnotetext{
${ }^{15} \mathrm{It}$ is assumed that atomic scale analysis, where individual charges may indeed be found and isolated, is not essential for explaining these microscopic electrodynamic phenomena.
} 


\subsubsection{Second stage of the experiment. A new split for $\mathbf{E}^{T O T}$.}

If the experiment is repeated with the same driving external magnetic field, but using an open circuit, the equation of continuity of electric charges, assumed valid in 4 of $\S 1.3$, allows predicting that charges will accumulate at the wire ends. As said, these will generate a conservative electric field, which:

- opposes to the induced nonconservative electric field inside the conductor, and

- contributes to the nonconservative electric field outside the conductor.

These two kinds of electric fields (conservative and nonconservative) will be defined and quantified separately before any further experimental results are modeled.

For this purpose, consider Coulomb's law, assumed valid in 9 of $\S 1.3$, which states that time-invariant charge distributions $\rho(\mathbf{x})$ generate conservative electrostatic fields $\mathbf{E}^{S T A T}(\mathbf{x})$ of the form:

$$
\mathbf{E}^{S T A T}(\mathbf{x})=\frac{1}{4 \pi \varepsilon_{0}} \int_{V^{\prime}} \rho\left(\mathbf{x}^{\prime}\right) \frac{\mathbf{x}-\mathbf{x}^{\prime}}{\left|\mathbf{x}-\mathbf{x}^{\prime}\right|^{3}} d V^{\prime}
$$

where $V^{\prime}$ is a spatial region large enough to contain all the charges that exert a measurable electric influence on a test particle located at $\mathbf{x}$, within experimental accuracy.

No assumption has been made that (3.4) would hold for time-varying fields.

Acknowledging the above, arbitrarily make a hypothetical extension of Coulomb's electrostatic law to time-varying fields and define $\mathbf{E}^{\rho}(t, \mathbf{x})$ as $^{16}$ :

\footnotetext{
${ }^{16}$ Some may object to this definition of $\mathbf{E}^{\rho}(t, \mathbf{x})$, in the sense that it would not represent a real physical field.

The integral in (3.5) requires the instantaneous contribution to the field at $\mathbf{x}$, of particles lying at $\mathbf{x}^{\prime}$, this is, located arbitrarily far from $\mathbf{x}$. How could any electric charge instantaneously transmit its influence through space? If the speed of light is limited, how could its constituent fields be expected to propagate with infinite speed? The concept of retarded potential, which proposes an answer to the above questions, renders equation (3.5) as describing a non-physical entity.

Should that be the case, the usage of non-physical fields for calculation purposes has been recommended by other authors (Faddeev-Popov, Schoenmaker et al.). On the other hand, it may be argued that such scope and usage is of a totally different nature and purpose as the one resulting from (3.5). (The Faddeev-Popov ghost fields are fictitious
} 


$$
\mathbf{E}^{\rho}(t, \mathbf{x})=\frac{1}{4 \pi \varepsilon_{0}} \int_{V^{\prime}} \rho\left(t, \mathbf{x}^{\prime}\right) \frac{\mathbf{x}-\mathbf{x}^{\prime}}{\left|\mathbf{x}-\mathbf{x}^{\prime}\right|^{3}} d V^{\prime}
$$

where $V^{\prime}$ is a spatial region large enough to contain all the charges that, if kept constant through time and equal to the largest value that they would exhibit throughout the experiment, would exert a measurable influence on a test particle located at $\mathbf{x}$, within experimental accuracy.

Call -as in the first stage of this experiment- $\mathbf{E}^{T O T}(t, \mathbf{x})$ the total electric field and define $\Delta \mathbf{E}(t, \mathbf{x})$ as:

$$
\mathbf{E}^{T O T}(t, \mathbf{x})=\mathbf{E}^{\rho}(t, \mathbf{x})+\Delta \mathbf{E}(t, \mathbf{x})
$$

As defined above, $\Delta \mathbf{E}(t, \mathbf{x})$ is the balance electric field.

In order to obtain the curl of $\mathbf{E}^{T O T}(t, \mathbf{x})$ and of its component fields, $\mathbf{E}^{\rho}(t, \mathbf{x})$ and $\Delta \mathbf{E}(t, \mathbf{x})$, first note that it is desirable that, -as in the static case- equation (3.5) defines $\mathbf{E}^{\rho}(t, \mathbf{x})$ as a gradient, by writing (3.5) as:

$$
\mathbf{E}^{\rho}(t, \mathbf{x})=-\frac{1}{4 \pi \varepsilon_{0}} \int_{V^{\prime}} \rho\left(t, \mathbf{x}^{\prime}\right) \nabla\left(\frac{1}{\left|\mathbf{x}-\mathbf{x}^{\prime}\right|}\right) d V^{\prime}
$$

or

$$
\mathbf{E}^{\rho}(t, \mathbf{x})=-\nabla\left[\frac{1}{4 \pi \varepsilon_{0}} \int_{V^{\prime}} \rho\left(t, \mathbf{x}^{\prime}\right)\left(\frac{1}{\left|\mathbf{x}-\mathbf{x}^{\prime}\right|}\right) d V^{\prime}\right]=-\nabla \phi^{\rho}(t, \mathbf{x})
$$

If $\mathbf{E}^{\rho}(t, \mathbf{x})$ is continuously differentiable in the region under analysis, then a necessary and sufficient condition for deriving (3.8) out of (3.7) is that $\mathbf{E}^{\rho}(t, \mathbf{x})$ be irrotational. So, let:

$$
\nabla \times \mathbf{E}^{\rho}(t, \mathbf{x})=\mathbf{0}
$$

In this manner, due to (3.6), the Maxwell-Faraday induction equation (3.1) predicts that:

anti-commuting complex scalar fields, introduced by their authors in the mid 70's for the quantization of non-Abelian gauge theories in order to formulate them as consistent quantum field theories [15]. Schoenmaker, Magnus and Meuris extended the concept for solving the classical field equations of a gauge theory in 2002 [16]).

It is not the intention of this work to enter into such controversy at this time, therefore momentaneously consider $\mathbf{E}^{\rho}(t, \mathbf{x})$ to be a mere mathematical construct, the proposition being that any differences between $\mathbf{E}^{\rho}(t, \mathbf{x})$ and the "physical electric field" should be taken into account in a complement field, $\Delta \mathbf{E}(t, \mathbf{x})$, as defined in equation (3.6). 


$$
\nabla \times \mathbf{E}^{T O T}(t, \mathbf{x})=\nabla \times\left(\mathbf{E}^{\rho}(t, \mathbf{x})+\Delta \mathbf{E}(t, \mathbf{x})\right)=-\frac{\partial \mathbf{B}^{T O T}(t, \mathbf{x})}{\partial t}
$$

where, applying (3.9),

$$
\nabla \times \Delta \mathbf{E}(t, \mathbf{x})=-\frac{\partial \mathbf{B}^{T O T}(t, \mathbf{x})}{\partial t}
$$

Having analyzed the curl of the electric field components $\mathbf{E}^{\rho}(t, \mathbf{x})$ and $\Delta \mathbf{E}(t, \mathbf{x})$, their divergence will be now studied.

From (3.5), the divergence of $\mathbf{E}^{\rho}(t, \mathbf{x})$ is:

$$
\nabla \cdot \mathbf{E}^{\rho}(t, \mathbf{x})=-\frac{1}{4 \pi \varepsilon_{0}} \int_{V^{\prime}} \rho\left(t, \mathbf{x}^{\prime}\right) \nabla^{2}\left(\frac{1}{\left|\mathbf{x}-\mathbf{x}^{\prime}\right|}\right) d V^{\prime}
$$

or

$$
\nabla \cdot \mathbf{E}^{\rho}(t, \mathbf{x})=\frac{1}{\varepsilon_{0}} \int_{V^{\prime}} \rho\left(t, \mathbf{x}^{\prime}\right) \delta\left(\mathbf{x}-\mathbf{x}^{\prime}\right) d V^{\prime}=\frac{1}{\varepsilon_{0}} \rho(t, \mathbf{x})
$$

Calculating the divergence of both sides in (3.6) and considering the Maxwell-Gauss equation (3.3) yields:

$$
\nabla \cdot \mathbf{E}^{T O T}(t, \mathbf{x})=\nabla \cdot \mathbf{E}^{\rho}(t, \mathbf{x})+\nabla \cdot \Delta \mathbf{E}(t, \mathbf{x})=\frac{\rho(t, \mathbf{x})}{\varepsilon_{0}}
$$

where, applying (3.13),

$$
\nabla \cdot \mathbf{E}^{T O T}(t, \mathbf{x})=\frac{\rho(t, \mathbf{x})}{\varepsilon_{0}}+\nabla \cdot \Delta \mathbf{E}(t, \mathbf{x})=\frac{\rho(t, \mathbf{x})}{\varepsilon_{0}}
$$

implying that:

$$
\nabla \cdot \Delta \mathbf{E}(t, \mathbf{x})=0
$$

Based on the conjecture that Coulomb's law may be mathematically extendable to time-varying fields, the total electrodynamic field $\mathbf{E}^{T O T}$ locally existing at any point (event) in spacetime has been mathematically partitioned into two theoretical component vector fields, $\mathbf{E}^{\rho}$ and $\Delta \mathbf{E}$. Unique, well defined, local and measurable attributes have been determined for these fields. As a consequence of this, each of these fields satisfies one Maxwell null condition, respectively bearing the full compliance owed by the total field with the other Maxwell condition, as shown in table 1 below: 


\begin{tabular}{|l|l|l|l|}
\hline & $\mathbf{E}^{\rho}(t, \mathbf{x})$ & $\Delta \mathbf{E}(t, \mathbf{x})$ & $\mathbf{E}^{T O T}(t, \mathbf{x})$ \\
\hline Divergence & $\frac{\rho(t, \mathbf{x})}{\varepsilon_{0}}$ & 0 & $\frac{\rho(t, \mathbf{x})}{\varepsilon_{0}}$ \\
\hline Curl & 0 & $-\frac{\partial \mathbf{B}^{T O T}(t, \mathbf{x})}{\partial t}$ & $-\frac{\partial \mathbf{B}^{T O T}(t, \mathbf{x})}{\partial t}$ \\
\hline $\begin{array}{l}\text { Energetic } \\
\text { behavior }\end{array}$ & Conservative & $\begin{array}{l}\text { Non } \\
\text { conservative }\end{array}$ & Combination \\
\hline
\end{tabular}

Table 1: Local Features of $\mathbf{E}^{\rho}$ and $\Delta \mathbf{E}$

Table 3.1: Local Features of $\mathbf{E}^{\rho}$ and $\Delta \mathbf{E}$

In this manner, given an electric field $\mathbf{E}^{T O T}(t, \mathbf{x})$ defined in all of spacetime, its divergence and curl (both unique, well defined, local and measurable physical attributes) could in principle be locally measured anywhere in its domain, and thereafter locally associated with the (local, as well) attributes of its component fields, $\mathbf{E}^{\rho}$ and $\Delta \mathbf{E}$ respectively. The everywhere local definition of these attributes is sufficient to uniquely define in turn the fields $\mathbf{E}^{\rho}$ and $\Delta \mathbf{E}$ throughout all spacetime.

This principle of operational calculus -that the local definition of a field induces in turn its global definition- was used by Lorentz [17] in the formulation of his microscopic version of Maxwell's electromagnetic field theory. Though Lorentz believed in retarded action ${ }^{17}$, the observations that motivated footnote 16 should -with this- be satisfactorily addressed and the conjecture, mathematically validated ${ }^{18}$.

Other partitions of the electric field have been historically used. For example, $\mathbf{E}^{T O T}$ has been split into its electrostatic and electrodynamic components, into its near, midrange and far-field components, or into its frequency spectrum, etc. The partition for $\mathbf{E}^{T O T}$ shown in table 1 above has the merit of being coherently aligned with the Maxwell equations. In effect: because continuously differentiable 3 -D vector fields are uniquely defined (except for a trivial term) by their curl and divergence, both Maxwell equations, (3.1) and (3.3), are needed in order to completely define $\mathbf{E}^{T O T}$. If only one equation is used, certain attributes of the electric field remain undefined. Given one Maxwell equation, (either (3.1) or (3.3)) the proposed partition assigns all the well-defined attributes to one field, (either $\Delta \mathbf{E}$ or

\footnotetext{
${ }^{17}$ In chapter IV of [17] Lorentz stresses -perhaps following a concept introduced by Gauss in 1845- that the action of one charged particle on another is propagated at the speed of light.

${ }^{18}$ Those still uneasy with this mathematical split of $\mathbf{E}^{T O T}$ into $\mathbf{E}^{\rho}$ and $\Delta \mathbf{E}$ may wish to refer to section 4.2 Discussion, part 1.
} 
$\mathbf{E}^{\rho}$ respectively), reserving all uncertainties for the other, and vice versa.

This is: due to (3.9),

$$
\nabla \times \Delta \mathbf{E}(t, \mathbf{x})=\nabla \times\left(\Delta \mathbf{E}(t, \mathbf{x})+\lambda_{1} \mathbf{E}^{\rho}(t, \mathbf{x})\right) \quad \forall \lambda_{1} \in R
$$

The above, when replaced in (3.10) yields:

$$
\nabla \times\left(\Delta \mathbf{E}(t, \mathbf{x})+\lambda_{1} \mathbf{E}^{\rho}(t, \mathbf{x})\right)=-\frac{\partial \mathbf{B}^{T O T}(t, \mathbf{x})}{\partial t} \quad \forall \lambda_{1} \in R
$$

Similarly, due to (3.16),

$$
\nabla \cdot \mathbf{E}^{\rho}(t, \mathbf{x})=\nabla \cdot\left(\mathbf{E}^{\rho}(t, \mathbf{x})+\lambda_{2} \Delta \mathbf{E}(t, \mathbf{x})\right) \quad \forall \lambda_{2} \in R
$$

which, when replaced in (3.13) yields:

$$
\nabla \cdot\left(\mathbf{E}^{\rho}(t, \mathbf{x})+\lambda_{2} \Delta \mathbf{E}(t, \mathbf{x})\right)=\frac{\rho(t, \mathbf{x})}{\varepsilon_{0}} \quad \forall \lambda_{2} \in R
$$

The infinite selection of values that $\lambda_{1}$ and $\lambda_{2}$ may adopt without altering the physical validity of equations (3.18) and (3.20) clearly represent the uncertainty contained in this formulation. Attention is drawn to the following facts:

1. This uncertainty -though detectable due to the way in which the $\mathbf{E}^{T O T}$ field was split- is not a consequence of such a split, but of the insufficient number of analytical equations under consideration.

2. Though in some ways similar to the gauges existing in the vector and scalar electrodynamic potentials, the uncertainty highlighted herein is of an essentially different nature. While the gauges alter the potentials in nonmensurable ways (and so are innocuous to the measurable electric and magnetic fields and their mutual physical relation), the span of mathematical options that $\lambda_{2}$ represents points directly to the slack present in the definition of measurable phenomena, calling -as will be seen in $\S 3.3 .1$ - for an experiment in order to determine the physically correct values and relations.

The acknowledgement of these facts will be essential in later stages of this work. 


\subsection{Currents}

\subsubsection{An expression for the divergence of $\mathbf{j}$.}

Having split $\mathbf{E}^{T O T}$ into its component fields, the current density $\mathbf{j}$ may now be analyzed. This step is necessary before deriving an expression for the magnetic field.

Equation (3.20) may be written as:

$$
\rho(t, \mathbf{x})=\varepsilon_{0} \nabla \cdot\left(\mathbf{E}^{\rho}(t, \mathbf{x})+\lambda_{2} \Delta \mathbf{E}(t, \mathbf{x})\right) \quad \forall \lambda_{2} \in R
$$

in such a way that the equation of continuity for electric charges (really an equation for the continuity of $\mathbf{j}$ ), assumed valid in 4 of $\S 1.3$,

$$
\nabla \cdot \mathbf{j}(t, \mathbf{x})+\frac{\partial \rho(t, \mathbf{x})}{\partial t}=0
$$

may, in turn be written as:

$(3.23) \nabla \cdot \mathbf{j}(t, \mathbf{x})+\frac{\partial}{\partial t}\left[\varepsilon_{0} \nabla \cdot\left(\mathbf{E}^{\rho}(t, \mathbf{x})+\lambda_{2} \Delta \mathbf{E}(t, \mathbf{x})\right)\right]=0 \quad \forall \lambda_{2} \in R$

or, swapping the spatial and temporal differential operators,

$(3.24) \nabla \cdot \mathbf{j}(t, \mathbf{x})+\nabla \cdot\left[\varepsilon_{0} \frac{\partial}{\partial t}\left(\mathbf{E}^{\rho}(t, \mathbf{x})+\lambda_{2} \Delta \mathbf{E}(t, \mathbf{x})\right)\right]=0 \quad \forall \lambda_{2} \in R$

this is, a mathematically complete equation for the continuity of the vector field $\mathbf{j}(t, \mathbf{x})$ reads:

$$
\nabla \cdot\left[\mathbf{j}(t, \mathbf{x})+\varepsilon_{0} \frac{\partial}{\partial t}\left(\mathbf{E}^{\rho}(t, \mathbf{x})+\lambda_{2} \Delta \mathbf{E}(t, \mathbf{x})\right)\right]=0 \quad \forall \lambda_{2} \in R
$$

\subsubsection{In search for a solenoidal current.}

Maxwell realized that, when non-solenoidal currents were considered, the relation known to exist between (conduction) currents and magnetic fields (i.e., Ampère's relation, which Maxwell calls "Equations of Electric Currents" and labels (E) in [18]) did not resist mathematical analysis. In his work [18], he says:

"By differentiating the equations ( $E$ ) with respect to $x, y$ and $z$, respectively and adding the results we obtain the equation 


$$
\frac{d u}{d x}+\frac{d v}{d y}+\frac{d w}{d z}=0 \quad 19
$$

which indicates that the current whose components are $u, v, w$ is subject to the condition of motion of an incompressible fluid, and that it must necessarily flow in closed circuits."

And, referring to the above described solenoidal feature of the current whose components are $u, v, w$, Maxwell concludes:

"This equation is true only if we take $u, v$ and $w$ as the components of that electric flow which is due to the variation of electric displacement as well as true conduction."

Formally declaring, a few paragraphs later in his work [19]:

"[610] One of the chief peculiarities of this treatise is the doctrine which it asserts, that the true electric current $\mathcal{C}$, that on which the electromagnetic phenomena depend, is not the same thing as $\mathcal{K}$, the current of conduction, but that the time variation of $\mathcal{D}$, the electric displacement must be taken into account in estimating the total movement of electricity, so that we must write,

$$
\mathcal{C}=\mathcal{K}+\dot{\mathcal{D}} \quad \text { (Equation of True Currents) } \quad(H) "
$$

It is clear from the above, that Maxwell modified what is now called Ampère's relation. It is also clear that he did so because he was in search for a solenoidal current term for it. This is, Maxwell was not concerned about correcting the expression in order to match a new experimental value or behavior recently reported by some respectable observer.

Maxwell himself declared not having modified Ampère's relation due to experimental, but theoretical reasons. In fact, only a few paragraphs before his above mentioned conclusions, he states in [20]:

"[606] Up to this point in our investigation, we have deducted everything from purely dynamical considerations, without any reference to quantitative experiments in electricity or

\footnotetext{
${ }^{19}$ Maxwell employs the sign reserved today for total derivatives, though he refers to partial derivatives. As Einstein says in his introduction to [5], these were the first uses of partial derivatives in the area of physics.
} 
magnetism. The only use we have made of experimental knowledge is to recognize, in the abstract quantities deduced from the theory, the concrete quantities discovered by experiment, and to denote them by names which indicate their physical relations rather that their mathematical generation."... "We have not, however, obtained any data for determining either $\mathcal{A}$ or $\mathcal{B}$ from the distribution of currents in the field. For this purpose we must find the mathematical connexion (sic) between these quantities and the currents."

The theoretical drive and lack of experimental verification in Maxwell's approach to this aspect of his electromagnetic field model will become an essential argument in this work, so one more evidence that Ampère's relation was subject to a theoretical (as opposed to experimental) modification is hereby offered: As Maxwell himself declared [18], the amount of experimental evidence available at the time, made any experimental corrections difficult or unfeasible:

"We have very little experimental evidence relating to the direct electromagnetic action of currents due to the variation of electric displacement in dielectrics, but the extreme difficulty of reconciling the laws of electromagnetism with the existence of currents which are not closed is one reason among many why we must admit the existence of transient currents due to the variation of displacement. Their importance will be seen when we come to the electromagnetic theory of light."

\subsection{The Magnetic Field}

\subsubsection{Exploring Maxwell's options.}

If Maxwell was merely looking for a solenoidal term for the Equation of Electric Currents by writing:

$$
V \nabla \mathcal{H}=4 \pi(\mathcal{K}+\dot{\mathcal{D}})
$$

where, expressing (3.26) in contemporaneous notation as Maxwell-Ampère's equation ${ }^{20}$ :

$$
\nabla \times \mathbf{B}^{T O T}=\mu_{0}\left(\mathbf{j}+\frac{\partial}{\partial t} \varepsilon_{0} \mathbf{E}^{T O T}\right)
$$

\footnotetext{
${ }^{20}$ Remember that this analysis is for a medium with $\mu_{0}, \varepsilon_{0}$.
} 
then, (3.25) shows that he could have obtained infinitely many more solenoidal terms for Maxwell-Ampère's expression by writing, instead of the expression in parenthesis of (3.27):

$$
\mathbf{j}(t, \mathbf{x})+\frac{\partial}{\partial t} \varepsilon_{0}\left(\mathbf{E}^{\rho}(t, \mathbf{x})+\lambda_{2} \Delta \mathbf{E}(t, \mathbf{x})\right) \quad \forall \lambda_{2} \in R
$$

Though every value of $\lambda_{2} \in R$ makes (3.28) satisfy a solenoidal requirement, only one instance of (3.28) is physically equal to the curl of $\mathbf{B}^{T O T}(t, \mathbf{x})$. This is so because, being $\mathbf{B}^{T O T}(t, \mathbf{x})$ a unique, well-behaved field $^{21}$, its curl is unique.

In order to determine which value of $\lambda_{2}$ produces the physically adequate instance of (3.28) and defines the exact form of Maxwell-Ampère's relation, experimental work is needed. This, because Faraday's, Ampère's, Biot-Savart's and Coulomb's equations summarize the results of experimental observations. As with all physical theories, they may be mathematically challenged, but must be experimentally formulated. This feature distinguishes the present work from traditional gauge theories, as anticipated in $\S 3.1 .2$.

As documented in the references above, Maxwell did not perform the necessary experiments and, by postulating (3.26) (this is, (3.27)), he inadvertently assumed the equivalent of an equation (3.28) in which $\lambda_{2}=1$.

Two facts probably led Maxwell to formulate (3.26):

1. Maxwell conceived the total current as composed of two parts: the conduction current $\mathcal{K}$, and the displacement current, due to the time variation of $\mathcal{D}$. He never conceived a naturally solenoidal term (such as $\left.\varepsilon_{0} \Delta \mathbf{E}\right)$ to be a constituent of the total current. Therefore, he had no null term to add to his proposition.

2. Given the historical development of the electromagnetic field model, equation (3.26) is a more "natural" choice than (3.28).

\subsubsection{The mathematical options for $B^{T O T}$.}

Consider the formula due to Biot and Savart, assumed valid in 8 of $\S 1.3$, which states that time-invariant currents $\mathbf{j}(\mathbf{x})$ generate magnetostatic fields $\mathbf{B}^{S T A T}(\mathbf{x})$ of the form:

$$
\mathbf{B}^{S T A T}(\mathbf{x})=\frac{\mu_{0}}{4 \pi} \int_{V^{\prime}} \mathbf{j}\left(\mathbf{x}^{\prime}\right) \times \frac{\mathbf{x}-\mathbf{x}^{\prime}}{\left|\mathbf{x}-\mathbf{x}^{\prime}\right|^{3}} d V^{\prime}
$$

\footnotetext{
${ }^{21}$ I.e., continuously differentiable.
} 
where $V^{\prime}$ is a spatial region large enough to contain all the currents that exert a measurable magnetic influence on a test circuit located at $\mathbf{x}$, within experimental accuracy. The Biot-Savart expression is equivalent to Ampère's original expression, assumed valid in 7 of $\S 1.3^{22}$.

No assumption has been made that (3.29) would apply to time-varying fields. So, in a manner totally equivalent to that used for the electric field case, arbitrarily ${ }^{23}$ define, for time-varying currents, the following magnetic field:

$$
\mathbf{B}^{j}(t, \mathbf{x})=\frac{\mu_{0}}{4 \pi} \int_{V^{\prime}} \mathbf{j}\left(t, \mathbf{x}^{\prime}\right) \times \frac{\mathbf{x}-\mathbf{x}^{\prime}}{\left|\mathbf{x}-\mathbf{x}^{\prime}\right|^{3}} d V^{\prime}
$$

where $V^{\prime}$ is a spatial region large enough to contain all the currents that, if kept constant through time and equal to the largest value that they would exhibit throughout the experiment, would exert a measurable influence on a test circuit located at $\mathbf{x}$ within experimental accuracy. Call $\mathbf{B}^{T O T}(t, \mathbf{x})$ the total magnetic field and further define $\Delta \mathbf{B}(t, \mathbf{x})$ as the balance magnetic field:

$$
\mathbf{B}^{T O T}(t, \mathbf{x})=\mathbf{B}^{j}(t, \mathbf{x})+\Delta \mathbf{B}(t, \mathbf{x})
$$

In order to analyze the divergence and curl of $\mathbf{B}^{T O T}$ and its component fields, first note that it is desirable to express $\mathbf{B}^{j}$ as a rotor, by writing (3.30) as follows:

$$
\mathbf{B}^{j}(t, \mathbf{x})=-\frac{\mu_{0}}{4 \pi} \int_{V^{\prime}} \mathbf{j}\left(t, \mathbf{x}^{\prime}\right) \times \nabla\left(\frac{1}{\left|\mathbf{x}-\mathbf{x}^{\prime}\right|}\right) d V^{\prime}
$$

or

$$
\mathbf{B}^{j}(t, \mathbf{x})=\frac{\mu_{0}}{4 \pi} \nabla \times \int_{V^{\prime}} \frac{\mathbf{j}\left(t, \mathbf{x}^{\prime}\right)}{\left|\mathbf{x}-\mathbf{x}^{\prime}\right|} d V^{\prime}=\nabla \times \mathbf{A}^{j}(t, \mathbf{x})
$$

Assuming that $\mathbf{B}^{j}$ is continuously differentiable in the region under analysis,

$$
\nabla \cdot \mathbf{B}^{j}(t, \mathbf{x})=\nabla \cdot \nabla \times \mathbf{A}^{j}(t, \mathbf{x})=0
$$

\footnotetext{
${ }^{22}$ In fact, the question of their suspected equivalence was finally and positively settled in arbitration by Laplace. The story says that in order to decide, Laplace required from Ampère to prove that, upon integration of his expression, Biot expression results. Succeeding in his demonstration, Ampère sent it -unpublished- to Laplace... and the interaction law was thereafter named "Law of Laplace"! [21].

${ }^{23}$ Comments equivalent to those for $\mathbf{E}^{\rho}$ in footnote 16 apply.
} 
On the other hand, respectable observers consistently report that magnetic monopoles have not yet been detected. So, until the existence of magnetic monopoles is in fact reported, a null Maxwell-Gauss assumption on $\mathbf{B}^{T O T}(t, \mathbf{x})$ allows predicting that $\nabla \cdot \mathbf{B}^{T O T}(t, \mathbf{x})=0$, leading to $\nabla \cdot \Delta \mathbf{B}(t, \mathbf{x})=0$.

In order to determine the curl of $\mathbf{B}^{T O T}$, first consider the curl of $\mathbf{B}^{j}(t, \mathbf{x})$, by applying the curl operator to (3.30) (or better, to (3.33)): ${ }^{24}$

$$
\begin{aligned}
& \nabla \times \mathbf{B}^{j}(t, \mathbf{x})=\frac{\mu_{0}}{4 \pi} \nabla \times\left(\nabla \times \int_{V^{\prime}} \frac{\mathbf{j}\left(t, \mathbf{x}^{\prime}\right)}{\left|\mathbf{x}-\mathbf{x}^{\prime}\right|} d V^{\prime}\right)= \\
& =\frac{\mu_{0}}{4 \pi}\left[-\int_{V^{\prime}} \mathbf{j}\left(t, \mathbf{x}^{\prime}\right) \nabla^{2}\left(\frac{1}{\left|\mathbf{x}-\mathbf{x}^{\prime}\right|}\right) d V^{\prime}+\int_{V^{\prime}}\left(\mathbf{j}\left(t, \mathbf{x}^{\prime}\right) \cdot \nabla^{\prime}\right) \nabla^{\prime}\left(\frac{1}{\left|\mathbf{x}-\mathbf{x}^{\prime}\right|}\right) d V^{\prime}\right]
\end{aligned}
$$

The first integral in the right hand side of (3.35) contains a Dirac delta. The second may be integrated by parts into:

$$
\begin{aligned}
& \int_{V^{\prime}}\left(\mathbf{j}\left(t, \mathbf{x}^{\prime}\right) \cdot \nabla^{\prime}\right) \nabla^{\prime}\left(\frac{1}{\left|\mathbf{x}-\mathbf{x}^{\prime}\right|}\right) d V^{\prime}= \\
& =\widehat{\mathbf{x}}_{k} \int_{V^{\prime}} \nabla^{\prime} \cdot\left\{\mathbf{j}\left(t, \mathbf{x}^{\prime}\right)\left[\frac{\partial}{\partial x_{k}^{\prime}}\left(\frac{1}{\left|\mathbf{x}-\mathbf{x}^{\prime}\right|}\right)\right]\right\} d V^{\prime}-\int_{V^{\prime}}\left[\nabla^{\prime} \cdot \mathbf{j}\left(t, \mathbf{x}^{\prime}\right)\right] \nabla^{\prime}\left(\frac{1}{\left|\mathbf{x}-\mathbf{x}^{\prime}\right|}\right) d V^{\prime}
\end{aligned}
$$

this is:

$$
\begin{aligned}
& \int_{V^{\prime}}\left(\mathbf{j}\left(t, \mathbf{x}^{\prime}\right) \cdot \nabla^{\prime}\right) \nabla^{\prime}\left(\frac{1}{\left|\mathbf{x}-\mathbf{x}^{\prime}\right|}\right) d V^{\prime}= \\
& =\widehat{\mathbf{x}}_{k} \int_{S^{\prime}}\left[\mathbf{j}\left(t, \mathbf{x}^{\prime}\right) \frac{\partial}{\partial x_{k}^{\prime}}\left(\frac{1}{\left|\mathbf{x}-\mathbf{x}^{\prime}\right|}\right)\right] \cdot d \mathbf{S}^{\prime}-\int_{V^{\prime}}\left[\nabla^{\prime} \cdot \mathbf{j}\left(t, \mathbf{x}^{\prime}\right)\right] \nabla^{\prime}\left(\frac{1}{\left|\mathbf{x}-\mathbf{x}^{\prime}\right|}\right) d V^{\prime}
\end{aligned}
$$

\footnotetext{
${ }^{24}$ The mathematics in (3.35) through (3.38) were obtained from Bo Thidé's book "Electromagnetic Field Theory", Upsilon Books, 1997, Uppsala University, Sweden [22].

There are differences though, between [22] and the work presented here. While Thidé assigns the results of these operations to the curl of $\mathbf{B}^{T O T}$, in this work they are equated to the curl of $\mathbf{B}^{j}$.
} 
The first integral in the right hand side of (3.37) vanishes when integrated over a large sphere $S^{\prime}$ far from $\mathbf{x}$. The second integral combines with the equation of continuity for electric charges (3.22) to yield:

$$
\begin{aligned}
& \int_{V^{\prime}}\left(\nabla^{\prime} \cdot \mathbf{j}\left(t, \mathbf{x}^{\prime}\right)\right) \nabla^{\prime}\left(\frac{1}{\left|\mathbf{x}-\mathbf{x}^{\prime}\right|}\right) d V^{\prime}= \\
& =-\int_{V^{\prime}} \frac{\partial \rho\left(t, \mathbf{x}^{\prime}\right)}{\partial t} \nabla^{\prime}\left(\frac{1}{\left|\mathbf{x}-\mathbf{x}^{\prime}\right|}\right) d V^{\prime}=-\frac{\partial}{\partial t} \int_{V^{\prime}} \rho\left(t, \mathbf{x}^{\prime}\right) \nabla^{\prime}\left(\frac{1}{\left|\mathbf{x}-\mathbf{x}^{\prime}\right|}\right) d V^{\prime}
\end{aligned}
$$

The definition of $\mathbf{E}^{\rho}(t, \mathbf{x})$ in (5) allows writing ${ }^{25}$ :

$$
\int_{V^{\prime}}\left(\nabla^{\prime} \cdot \mathbf{j}\left(t, \mathbf{x}^{\prime}\right)\right) \nabla^{\prime}\left(\frac{1}{\left|\mathbf{x}-\mathbf{x}^{\prime}\right|}\right) d V^{\prime}=-4 \pi \varepsilon_{0} \frac{\partial}{\partial t} \mathbf{E}^{\rho}(t, \mathbf{x})
$$

In this manner, the curl of $\mathbf{B}^{j}(t, \mathbf{x})$ is uniquely given by:

$$
\begin{aligned}
& \nabla \times \mathbf{B}^{j}(t, \mathbf{x})= \\
& =\mu_{0} \int_{V^{\prime}} \mathbf{j}\left(t, \mathbf{x}^{\prime}\right) \delta\left(\mathbf{x}-\mathbf{x}^{\prime}\right) d V^{\prime}+\mu_{0} \varepsilon_{0} \frac{\partial \mathbf{E}^{\rho}(t, \mathbf{x})}{\partial t}=\mu_{0}\left(\mathbf{j}(t, \mathbf{x})+\varepsilon_{0} \frac{\partial \mathbf{E}^{\rho}(t, \mathbf{x})}{\partial t}\right)
\end{aligned}
$$

It is interesting to verify that $\mathbf{B}^{j}(t, \mathbf{x})$ and its curl are both well defined in (3.30) and (3.40), irrespective of whether $\mathbf{j}(t, \mathbf{x})$ is solenoidal or not. In effect; if $\mathbf{B}^{j}(t, \mathbf{x})$ is defined in (3.30) as the application of Biot-Savart on the solenoidal current

$$
\mathbf{j}(t, \mathbf{x})+\varepsilon_{0} \frac{\partial \mathbf{E}^{\rho}(t, \mathbf{x})}{\partial t}
$$

instead of the current $\mathbf{j}(t, \mathbf{x})$ alone, expression (3.40) for the curl of $\mathbf{B}^{j}(t, \mathbf{x})$ remains unaltered ${ }^{26}$. In this respect, the Biot-Savart equation seems to be more powerful than Ampère's relation, since the former is directly extensible to non-solenoidal currents, mathematically calling for the need to introduce the time variation of an electric field, as envisioned by Maxwell.

\footnotetext{
${ }^{25}$ Bo Thidé makes the assumption that the last integral of (3.38) equates to $-4 \pi \varepsilon_{0} \mathbf{E}^{T O T}$ instead of $-4 \pi \varepsilon_{0} \mathbf{E}^{\rho}$. This gives $\Delta \mathbf{E}$ a "free ride" into the traditional expression for the curl of $\mathbf{B}$.

${ }^{26}$ In such case, the factor of the Dirac delta in (3.35) is $\mathbf{j}+\varepsilon_{0} \frac{\partial \mathbf{E} \rho}{\partial t}$, while the divergence in the second integral of the right-hand-side of (3.37) is null.
} 
Having obtained an expression for the curl of $\mathbf{B}^{j}(t, \mathbf{x})$, the curl of $\Delta \mathbf{B}(t, \mathbf{x})$ should now follow. Noting that Maxwell-Ampère's equation is a statement about the curl of $\mathbf{B}^{T O T}(t, \mathbf{x})$, (which includes the curl of $\Delta \mathbf{B}(t, \mathbf{x}))$, Maxwell-Ampère's equation will be analyzed.

For this, three aspects must be considered:

1. The first aspect to consider is that Maxwell's argument on nonsolenoidal conduction currents and their effects on the magnetic field is addressed -and totally satisfied- by (3.40).

2. A second aspect to consider is that $\nabla \cdot \Delta \mathbf{B}=0$, so unless null $\Delta \mathbf{B}$ fields are acceptable (which means accepting that the extension of BiotSavart to time-varying fields is not only a mathematical construct but a physical relation), $\Delta \mathbf{B}$ must exhibit a nonzero curl.

3. The third aspect to consider is Maxwell's model development strategy:

As already seen, Maxwell modified the Equation of Electric Currents

$$
\nabla \times \mathbf{B}^{T O T}(t, \mathbf{x})=\mu_{0} \mathbf{j}(t, \mathbf{x})
$$

to read

$$
\nabla \times \mathbf{B}^{T O T}(t, \mathbf{x})=\mu_{0} \mathbf{j}(t, \mathbf{x})+\mu_{0} \varepsilon_{0} \frac{\partial \mathbf{E}^{T O T}(t, \mathbf{x})}{\partial t}
$$

in order to satisfy the mathematical requirement that rotors of continuously derivable fields must be solenoidal.

But, equations (3.26) through (3.28) show that Maxwell had more mathematical options than those he used to fit the purpose when introducing the modification described in 3 above. Exploring these options will reveal to be of paramount importance.

So -following Maxwell's development strategy indicated in 3 above- the curl of $\mathbf{B}^{T O T}(t, \mathbf{x})$ will be assumed to equate to some solenoidal combination of fields satisfying the established assumptions, of which $\mathbf{j}(t, \mathbf{x})$ is a component. The infinite options for such solenoidal combinations of fields are given by equation (3.28), so that a complete mathematical reformulation of Maxwell's proposal should be given by: 


$$
\begin{aligned}
\nabla & \times \mathbf{B}^{T O T}(t, \mathbf{x})= \\
& =\mu_{0}\left[\mathbf{j}(t, \mathbf{x})+\varepsilon_{0} \frac{\partial}{\partial t}\left(\mathbf{E}^{\rho}(t, \mathbf{x})+\lambda_{2} \Delta \mathbf{E}(t, \mathbf{x})\right)\right] \quad \forall \lambda_{2} \in R
\end{aligned}
$$

Equation (3.41) makes it evident that Maxwell's solenoidal requirement, though sufficient for determining the unsuitability of Ampère's original equation, is not sufficient for determining its final form. As anticipated in section 3.3.1, from a physical standpoint there should be one and only one value of $\lambda_{2}$ and instance of (3.41) that agree with experimental data. Acknowledging this, Maxwell's proposition should have been:

$$
\begin{aligned}
\nabla & \times \mathbf{B}^{T O T}(t, \mathbf{x})= \\
& =\mu_{0}\left[\mathbf{j}(t, \mathbf{x})+\varepsilon_{0} \frac{\partial}{\partial t}\left(\mathbf{E}^{\rho}(t, \mathbf{x})+\lambda_{2} \Delta \mathbf{E}(t, \mathbf{x})\right)\right] \quad \text { for some } \lambda_{2} \in R
\end{aligned}
$$

As said, exactly which value of $\lambda_{2}$ gives Maxwell-Ampère's equation its definite form must be experimentally determined ${ }^{27}$.

An expression for the curl of $\Delta \mathbf{B}(t, \mathbf{x})$ may now be obtained, by introducing (3.31) and (3.40) into (3.42) and simplifying terms:

$$
\nabla \times \Delta \mathbf{B}(t, \mathbf{x})=\lambda_{2} \mu_{0} \varepsilon_{0} \frac{\partial \Delta \mathbf{E}(t, \mathbf{x})}{\partial t} \quad \text { for some } \lambda_{2} \in R
$$

Table 2 below summarizes the results obtained so far: the proposed split for $\mathbf{B}^{T O T}(t, \mathbf{x})$, and the features of each term:

\begin{tabular}{|l|l|l|l|}
\hline & $\mathbf{B}^{j}(t, \mathbf{x})$ & $\Delta \mathbf{B}(t, \mathbf{x})$ & $\mathbf{B}^{T O T}(t, \mathbf{x})$ \\
\hline Div. & 0 & 0 & 0 \\
\hline Curl & $\mu_{0} \mathbf{j}+\mu_{0} \varepsilon_{0} \frac{\partial \mathbf{E}^{\rho}(t, \mathbf{x})}{\partial t}$ & $\lambda_{2} \mu_{0} \varepsilon_{0} \frac{\partial \Delta \mathbf{E}(t, \mathbf{x})}{\partial t}$ & To be experi- \\
& & for some $\lambda_{2}$ to & mentally \\
& & be experimen- & determined \\
& & tally & \\
& & determined & \\
\hline
\end{tabular}

Table 2: Local Features of $\mathbf{B}^{j}$ and $\Delta \mathbf{B}$

These results, in conjunction with those of table 1 , constitute the findings of this work.

\footnotetext{
${ }^{27}$ As of today, no reports are known to the author on experiments aimed at determining such value of $\lambda_{2}$.
} 


\section{EPILOGUE}

\subsection{Conclusions.}

1. Maxwell was right in detecting that the equations describing the electromagnetic phenomena prevailing at that time were mathematically inconsistent and required a revision.

2. Maxwell's proposition, which resulted in a modification of Ampère's original equation for the case of time-varying fields, was incomplete. A complete, mathematically consistent revision of the prevailing set of equations describing the electromagnetic phenomena is:

From (3.18):

$$
\nabla \times\left(\lambda_{1} \mathbf{E}^{\rho}+\Delta \mathbf{E}\right)=-\frac{\partial \mathbf{B}^{T O T}}{\partial t} \quad \forall \lambda_{1} \in R
$$

From (3.20):

$$
\nabla \cdot\left(\mathbf{E}^{\rho}+\lambda_{2} \Delta \mathbf{E}\right)=\frac{\rho}{\varepsilon_{0}} \quad \forall \lambda_{2} \in R \quad(a)
$$

From experimental observations:

$$
\nabla \cdot \mathbf{B}^{T O T}=0
$$

From (3.41):

$$
\nabla \times \mathbf{B}^{T O T}=\mu_{0}\left[\mathbf{j}+\varepsilon_{0} \frac{\partial}{\partial t}\left(\mathbf{E}^{\rho}+\lambda_{2} \Delta \mathbf{E}\right)\right] \quad \forall \lambda_{2} \in R
$$

3. The scalar parameters $\lambda_{1}$ and $\lambda_{2}$ generate a family of candidate electromagnetic field models, all the members of which are mathematically consistent with the originating set of assumptions and therefore, from a mathematical point of view, undistinguishable.

4. Not all the members of the above family are physically adequate electromagnetic field models, as neither are all values of the scalar parameters. The physically correct models, jointly with the corresponding set of proper parameter values, are to be experimentally determined, leaving the last equation above as: 
From (3.42):

$$
\nabla \times \mathbf{B}^{T O T}=\mu_{0}\left[\mathbf{j}+\varepsilon_{0} \frac{\partial}{\partial t}\left(\mathbf{E}^{\rho}+\lambda_{2} \Delta \mathbf{E}\right)\right] \text { for some } \lambda_{2} \in R
$$

\subsection{Discussion.}

1. Those still unsatisfied with the definitions of $\mathbf{E}^{\rho}$ and $\mathbf{B}^{j}$, and the splitting that these definitions induce in the total electromagnetic fields $\mathbf{E}^{T O T}$ and $\mathbf{B}^{T O T}$, may wish to consider the following argument:

Let $\mathcal{L}$ be the family of all real valued scalar fields defined in spacetime which are Lebesgue integrable, and $\mathcal{C}^{1}$ the family of all real valued differentiable vector fields defined in spacetime. Let $\mathcal{G}$ be the subset of all gradient vector fields in $\mathcal{C}^{1}$.

Equation (3.5) defines an operator from $\mathcal{L}$ to $\mathcal{G}$. This operator univocally assigns to every $\rho(t, \mathbf{x}) / \varepsilon_{0}$ in $\mathcal{L}$, an irrotational (gradient) vector field $\mathbf{E}^{\rho}(t, \mathbf{x})$, in $\mathcal{G}$. Call this operator Coul, for Coulomb.

Reciprocally, given a vector field $\mathbf{E}(t, \mathbf{x})$ in $\mathcal{G}$, the div operator univocally assigns to it, its divergence $\nabla \cdot \mathbf{E}(t, \mathbf{x})$, which is in $\mathcal{L}$.

Between $\mathcal{L}$ and $\mathcal{G}$, the Coul and div operators form a mutually inverse couple. In effect, equation (3.13) shows that:

$$
\operatorname{div}\left[\operatorname{Coul}\left(\frac{\rho(t, \mathbf{x})}{\varepsilon_{0}}\right)\right]=\frac{\rho(t, \mathbf{x})}{\varepsilon_{0}}
$$

Applying Coul to both sides of $(c)$ :

$$
\operatorname{Coul}\left\{\operatorname{div}\left[\operatorname{Coul}\left(\frac{\rho(t, \mathbf{x})}{\varepsilon_{0}}\right)\right]\right\}=\operatorname{Coul}\left(\frac{\rho(t, \mathbf{x})}{\varepsilon_{0}}\right)
$$

But, $\operatorname{Coul}\left(\rho(t, \mathbf{x}) / \varepsilon_{0}\right)=\mathbf{E}^{\rho}(t, \mathbf{x})$, so :

$$
\operatorname{Coul}\left\{\operatorname{div}\left[\mathbf{E}^{\rho}(t, \mathbf{x})\right]\right\}=\mathbf{E}^{\rho}(t, \mathbf{x})
$$

Therefore, $(c)$ and $(d)$ above imply that:

$$
\text { Coul } \equiv d i v^{-1}
$$


The reciprocally inverse relation existing between div and Coul guarantees that, for every local (microscopic, or differential) definition of $\mathbf{E}^{\rho}$ (in terms of its divergence), a unique global (macroscopic, or integral) definition (in terms of Coul) exists, and vice versa. In other words, if a local definition of a field $\mathbf{E}^{\rho}$ is feasible, then a global definition of $\mathbf{E}^{\rho}$ is feasible as well. The global definition for the field $\mathbf{E}^{\rho}$ (locally defined in table 1 ) is given in (3.5).

A similar analysis may be made for $\mathbf{B}^{j}$ and $\mathbf{j}+\varepsilon_{0} \dot{\mathbf{E}}^{\rho}$.

These properties validate the differential formulation of integral fields, i.e., in the form of the well-known differential Maxwell field equations.

2. The value $\lambda_{1}$ appearing in equations (3.17) and (3.18), is not mentioned in table 1 or table 2 because the fields $\mathbf{E}^{\rho}$ and $\Delta \mathbf{E}$ are being treated there in a separate manner. The parameter $\lambda_{1}$ gains importance when both electric fields combine into one physical entity, as follows:

From (3.18) :

$$
\nabla \times\left(\mathbf{E}^{\rho}+\frac{1}{\lambda_{1}} \Delta \mathbf{E}\right)=-\frac{1}{\lambda_{1}} \frac{\partial \mathbf{B}^{T O T}}{\partial t} \quad \forall \lambda_{1} \neq 0 \in R
$$

So, if $\lambda_{1}$ is defined as $\lambda_{1}=\lambda_{2}^{-1} \quad \lambda_{1}, \lambda_{2} \neq 0^{28}$

and a new electric field $\boldsymbol{\Xi}$ is defined, as

$$
\boldsymbol{\Xi}(t, \mathbf{x})=\mathbf{E}^{\rho}(t, \mathbf{x})+\lambda_{2} \Delta \mathbf{E}(t, \mathbf{x}) \quad \text { for some } \lambda_{2} \neq 0 \in R
$$

then, for such value $\lambda_{2}$, equations $(a)$ above read:

$$
\begin{array}{ll}
\nabla \times \boldsymbol{\Xi} & =-\lambda_{2} \frac{\partial \mathbf{B}^{T O T}}{\partial t} \\
\nabla \cdot \boldsymbol{\Xi} & =\frac{\rho}{\varepsilon_{0}} \\
\nabla \cdot \mathbf{B}^{T O T} & =0 \\
\nabla \times \mathbf{B}^{T O T} & =\mu_{0}\left(\mathbf{j}+\varepsilon_{0} \frac{\partial \boldsymbol{\Xi}}{\partial t}\right)
\end{array}
$$

The field equations $(f)$ describe the dynamic behavior of $\boldsymbol{\Xi}$.

${ }^{28}$ The case $\lambda_{2}=0$ will be tretaed in point 3 of this $\S 4.2$ 
3. One trivial possibility for equation (3.42) is given by $\lambda_{2}=0$. However, since this makes $\nabla \times \Delta \mathbf{B}(t, \mathbf{x})=\mathbf{0}$ (and on the other hand, it is mathematically inferable from experimental observations that $\nabla \cdot \Delta \mathbf{B}(t, \mathbf{x})=0)$, this solution would force null $\Delta \mathbf{B}(t, \mathbf{x})$ fields, destroying the mutual coupling known to exist between electric and magnetic fields in free space ${ }^{29}$.

Another possibility is the traditional value $\lambda_{2}=1$-implicitly assumed by Maxwell, as described herein- which makes $\mathbf{\Xi}(t, \mathbf{x}) \equiv \mathbf{E}^{T O T}(t, \mathbf{x})$ and turns the reformulated field equations into the classical Maxwell field equations, leading to TSR, TGR and the claimed axiomatic conflict.

A third interesting possibility is the value $\lambda_{2}=-1$, which makes the reformulated equations invariant before Euclidean frame transformations, and gives the field $\boldsymbol{\Xi}$ the following physical interpretation:

Consider a microscopic analysis of the experiment in $\S 2$. An enlarged view of the setup in figure 2 , close to one of the discontinuity points (where the wire has been cut), reveals an accumulation of charges due to the action of the induced nonconservative field $\Delta \mathbf{E}(t, \mathbf{x})$. This accumulation of charges generates the conservative field $\mathbf{E}^{\rho}(t, \mathbf{x})$, which opposes the nonconservative field (tending to neutralize it) inside the conductor, while contributing to it in the exterior, increasing its effect. Figure 3 below schematizes this setup, where the origin of the $\mathrm{x}$-axis has been set at the selected discontinuity point, (where the conductor has been cut), with negative x-values and the conducting medium being represented to the left, while positive $\mathrm{x}$-values and vacuum, to the right:

\footnotetext{
${ }^{29}$ Said coupling would be limited exclusively to that provided by Ohm's law and the equation of continuity for electric charges (both relations operate only in the presence of matter).
} 


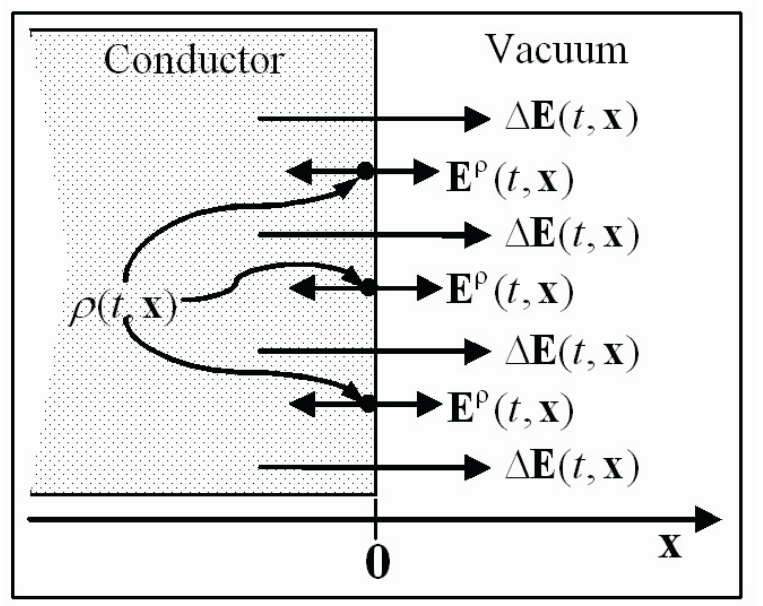

Figure 3 : Details of fields at the circuit discontinuity points

Symmetry considerations in the modelling of $\mathbf{E}^{\rho}$ lead to the conclusion that:

$$
\mathbf{E}^{\rho}\left(t, \mathbf{0}^{-}\right)=-\mathbf{E}^{\rho}\left(t, \mathbf{0}^{+}\right) \quad(g)
$$

while the solenoidal feature of $\Delta \mathbf{E}$ allows writing:

$$
\Delta \mathbf{E}\left(t, \mathbf{0}^{-}\right)=\Delta \mathbf{E}\left(t, \mathbf{0}^{+}\right)
$$

Adding equations $(g)$ and $(h)$ above leads to

$$
\mathbf{E}^{T O T}\left(t, \mathbf{0}^{-}\right)=-\mathbf{\Xi}\left(t, \mathbf{0}^{+}\right)
$$

while subtracting $(h)$ from $(g)$ leads to

$$
\boldsymbol{\Xi}\left(t, \mathbf{0}^{-}\right)=-\mathbf{E}^{T O T}\left(t, \mathbf{0}^{+}\right)
$$

Equations $(i)$ and $(j)$ provide the boundary conditions for equations $(f)$ when $\lambda_{2}=-1$.

4. The proposed field partition gives a new twist to one of the methods traditionally used for solving the electromagnetic field equations.

The electromagnetic field equations, together with the appropriate constitutive relations linking $\rho, \mathbf{j}$ and the fields, plus the initial and boundary conditions pertinent to the physical phenomenon under description, form a well-posed system of partial differential equations 
which completely determine $\mathbf{E}$ and $\mathbf{B}$. Notwithstanding, when generating solutions for the Maxwell field equations, the employment of a scalar potential $\phi$ and a vector potential $\mathbf{A}$ have been frequently preferred. As will be discussed herein, the method of electromagnetic potentials maps the solution space of the well-posed system of electromagnetic field equations into a new solution space (the space of potentials) that has the merit of exhibiting additional degrees of freedom respect the space of physical fields, freedom which is later used to the method's advantage by means of the introduction of the well-known electromagnetic gauges.

The method of electromagnetic potentials begins by noting that $\nabla \cdot \mathbf{B}=0$, which allows inferring that some real, differentiable vector field $\mathbf{A}(t, \mathbf{x})$ should exist, such that:

$$
\mathbf{B}(t, \mathbf{x})=\nabla \times \mathbf{A}(t, \mathbf{x})
$$

If the above expression for the magnetic field $\mathbf{B}$ is introduced in the Maxwell-Faraday field equation, a definition for the electric field $\mathbf{E}$, in terms of the electromagnetic potentials, is obtained:

$$
\mathbf{E}(t, \mathbf{x})=-\nabla \phi(t, \mathbf{x})-\dot{\mathbf{A}}(t, \mathbf{x})
$$

But equations $(k)$ and $(l)$ can also be considered as "partial definitions" for the vector potential A and scalar potential $\phi$, respectively. They are said to be partial because:

(a) a value for $\nabla \cdot \mathbf{A}(t, \mathbf{x})$ is missing in $(k)$, and

(b) since the missing divergence of $\mathbf{A}$ is a function of time, the time dependence of $\mathbf{A}$ has not been fully defined. This means that the gradient of $\phi$ has not been fully defined either in $(l)$, and therefore both potentials allow a certain freedom or "slack" in their definition, with the proviso that said slack be canceled when the potentials are related as in $(k)$ and $(l)^{30}$

\footnotetext{
${ }^{30}$ Giving rise to the concept of "gauge function" $\Gamma$ which, given one suitable pair of potentials $\phi$ and $\mathbf{A}$, enables spanning the complete set of suitable scalar and vector
} potentials by respectively setting, for any real, differentiable scalar field $\Gamma$ :

$$
\phi^{\prime}=\phi+\frac{\partial \Gamma}{\partial t} \quad \mathbf{A}^{\prime}=\mathbf{A}-\nabla \Gamma .
$$


In this work, the above potentials will be called "TOTAL" potentials, and will be partitioned into two components each (adopting for the potentials the nomenclature used in previous sections for the fields), so that:

$$
\phi^{T O T}(t, \mathbf{x})=\phi^{\rho}(t, \mathbf{x})+\Delta \phi(t, \mathbf{x})
$$

and

$$
\mathbf{A}^{T O T}(t, \mathbf{x})=\mathbf{A}^{j}(t, \mathbf{x})+\Delta \mathbf{A}(t, \mathbf{x})
$$

As above defined, $\Delta \phi$ and $\Delta \mathbf{A}$ will be respectively called the balance scalar potential and the balance vector potential. Then:

From equation (3.8):

$$
\mathbf{E}^{\rho}(t, \mathbf{x})=-\nabla \phi^{\rho}(t, \mathbf{x})
$$

so that the balance conditions to be satisfied in order to comply with (l) become:

$$
\Delta \mathbf{E}(t, \mathbf{x})=-\nabla \Delta \phi(t, \mathbf{x})-\frac{\partial \mathbf{A}^{j}(t, \mathbf{x})}{\partial t}-\frac{\partial \Delta \mathbf{A}(t, \mathbf{x})}{\partial t} \quad(m)
$$

Likewise, from equation (3.33):

$$
\mathbf{B}^{j}(t, \mathbf{x})=\nabla \times \mathbf{A}^{j}(t, \mathbf{x})
$$

so that the balance conditions to be satisfied in order to comply with (k) become:

$$
\Delta \mathbf{B}(t, \mathbf{x})=\nabla \times \Delta \mathbf{A}(t, \mathbf{x}) \quad(n)
$$

By using equations (3.8) and (3.33) as definitions for $\phi^{\rho}$ and $\mathbf{A}^{j}$ respectively, these components of the electromagnetic potentials become totally defined, and any slack existing in the definition of the total electromagnetic potentials is transferred to the balance components $\Delta \phi$ and $\Delta \mathbf{A}$. The concept of gauge, which has given rise to an entire branch of modern physics ${ }^{31}$ may then be associated in a more precise manner, not with the total electromagnetic potentials

\footnotetext{
${ }^{31}$ The origin of the standard model may be traced back to 1926 , when V. Fock applied the gauge concept to the quantum mechanics of charged particles that interact with electromagnetic fields.[23], [24].
} 
and fields, but with with specific components of them: the balance vector and balance scalar potentials, which generate the balance electromagnetic fields.

So, applying the findings of table 1 to the divergence of $(m)$,

$$
\nabla \cdot \Delta \mathbf{E}(t, \mathbf{x})=-\nabla^{2} \Delta \phi(t, \mathbf{x})-\nabla \cdot \frac{\partial \mathbf{A}^{j}(t, \mathbf{x})}{\partial t}-\nabla \cdot \frac{\partial \Delta \mathbf{A}(t, \mathbf{x})}{\partial t}=0
$$

where, adding and subtracting $\lambda_{2} \mu_{0} \varepsilon_{0} \Delta \ddot{\phi}(t, \mathbf{x})$ and rearranging terms,

$$
\begin{aligned}
\nabla^{2} \Delta \phi(t, \mathbf{x}) & -\lambda_{2} \mu_{0} \varepsilon_{0} \frac{\partial^{2} \Delta \phi(t, \mathbf{x})}{\partial t^{2}} \\
& +\frac{\partial}{\partial t}\left(\lambda_{2} \mu_{0} \varepsilon_{0} \frac{\partial \Delta \phi(t, \mathbf{x})}{\partial t}+\nabla \cdot \Delta \mathbf{A}(t, \mathbf{x})\right) \\
& =-\nabla \cdot \frac{\partial}{\partial t} \mathbf{A}^{j}(t, \mathbf{x}) \quad \text { for some } \lambda_{2} \in R
\end{aligned}
$$

On the other hand, applying the findings of table 2 to the curl of $(n)$,

$$
\begin{aligned}
\nabla \times \Delta \mathbf{B}(t, \mathbf{x}) & \equiv \\
& \equiv \nabla \times \nabla \times \Delta \mathbf{A}(t, \mathbf{x}) \\
& \equiv \nabla(\nabla \cdot \Delta \mathbf{A}(t, \mathbf{x}))-\nabla^{2} \Delta \mathbf{A}(t, \mathbf{x}) \\
& =\lambda_{2} \mu_{0} \varepsilon_{0} \frac{\partial \Delta \mathbf{E}(t, \mathbf{x})}{\partial t} \quad \text { for some } \lambda_{2} \in R
\end{aligned}
$$

or

$\nabla^{2} \Delta \mathbf{A}(t, \mathbf{x})+\lambda_{2} \mu_{0} \varepsilon_{0} \frac{\partial \Delta \mathbf{E}(t, \mathbf{x})}{\partial t}-\nabla(\nabla \cdot \Delta \mathbf{A}(t, \mathbf{x}))=0$ for some $\lambda_{2} \in R$ where, applying $(m)$ and rearranging terms, 


$$
\begin{aligned}
\nabla^{2} \Delta \mathbf{A}(t, \mathbf{x}) & -\lambda_{2} \mu_{0} \varepsilon_{0} \frac{\partial^{2} \Delta \mathbf{A}(t, \mathbf{x})}{\partial t^{2}} \\
& -\nabla\left(\lambda_{2} \mu_{0} \varepsilon_{0} \frac{\partial \Delta \phi(t, \mathbf{x})}{\partial t}+\nabla \cdot \Delta \mathbf{A}(t, \mathbf{x})\right) \\
& =\lambda_{2} \mu_{0} \varepsilon_{0} \frac{\partial^{2} \mathbf{A}^{j}(t, \mathbf{x})}{\partial t^{2}} \quad \text { for some } \lambda_{2} \in R
\end{aligned}
$$

The traditional solution procedure works similarly with $\phi^{T O T}, \mathbf{A}^{T O T}$ and the Maxwell field equations, to arrive to relations equivalent to $(o)$ and $(p)$, but posed for the total fields instead of the balance fields. At this stage, the traditional procedure makes use of the fact that $\nabla \cdot \mathbf{A}^{T O T}$ has not been defined, in order to simplify and uncouple the said equations into the Helmholtz wave equations by introducing the condition known as Lorentz gauge, which nullifies the terms between parentheses in the classical equivalents of $(o)$ and $(p)^{32}$.

On the other hand, the balance potentials $\Delta \phi$ and $\Delta \mathbf{A}$ (and their respective physical fields $\Delta \mathbf{E}$ and $\Delta \mathbf{B}$ ) are not constrained in any way by the equation of continuity of electric charges. In this manner, the slack in the definition of $\mathbf{A}^{T O T}$ and $\phi^{T O T}$ fully translates into a slack in the definitions of $\Delta \phi$ and $\Delta \mathbf{A}$, which may be used at will. For example, by means of the introduction of electromagnetic gauges. Some gauges which can be incorporated in this analysis are ${ }^{33}$ :

- The temporal, or Hamilton gauge: $\Delta \phi=0$.

- The radiation or transverse gauge: $\nabla \cdot \Delta \mathbf{A}=0$.

- The Coulomb gauge: $\Delta \phi=0$ and $\nabla \cdot \Delta \mathbf{A}=0$.

- The axial gauge: $\Delta A_{3}=0$.

\footnotetext{
${ }^{32}$ The Lorentz gauge (discovered by the Danish Physicist Ludwig Lorenz in 1867, decades before Lorentz demonstrated the covariance of Maxwell's equations) is no other than a different way of expressing the equation of continuity of electric charges and is furthermore, totally satisfied by the potentials $\phi^{\rho}$ and $\mathbf{A}^{j}$-completely and uniquely defined by (3.8) and (3.33) - in such a way that, when incorporated into the traditional method of electromagnetic potentials, it turns the equations of the traditional method into $(o)$ and $(p)$.

${ }^{33}$ The names used above correspond to an informal extension of the original nomenclature, as they have been borrowed from the classical gauge definitions, in terms of the total fields, $\phi^{T O T}$ and $\mathbf{A}^{T O T}$.
} 
One way of using the available slack is by arbitrarily extending ${ }^{34}$ the application of the Lorentz gauge to the balance potentials, so that the terms between parentheses in equations $(o)$ and $(p)$ are nullified. In this manner, the Helmholtz equations for the balance potentials become:

$$
\begin{aligned}
\nabla^{2} \Delta \phi(t, \mathbf{x}) & -\lambda_{2} \mu_{0} \varepsilon_{0} \frac{\partial^{2} \Delta \phi(t, \mathbf{x})}{\partial t^{2}} \\
& =-\nabla \cdot\left(\frac{\partial \mathbf{A}^{j}(t, \mathbf{x})}{\partial t}\right) \quad \text { for some } \lambda_{2} \in R
\end{aligned}
$$

and

$$
\begin{aligned}
\nabla^{2} \Delta \mathbf{A}( & t, \mathbf{x})-\lambda_{2} \mu_{0} \varepsilon_{0} \frac{\partial^{2} \Delta \mathbf{A}(t, \mathbf{x})}{\partial t^{2}} \\
& =\lambda_{2} \mu_{0} \varepsilon_{0} \frac{\partial}{\partial t}\left(\frac{\partial \mathbf{A}^{j}(t, \mathbf{x})}{\partial t}\right) \quad \text { for some } \lambda_{2} \in R
\end{aligned}
$$

Equations $(q)$ and $(r)$ make it evident that, while $\mathbf{E}^{\rho}$ and $\mathbf{B}^{j}$ are inextricably linked to their material (and therefore, localized) driving sources through the instantaneous analytical definitions of first order in the spatial coordinates (3.8) and (3.33), their balance counterparts $\Delta \mathbf{E}$ and $\Delta \mathbf{B}$ are related to potentials the behavior of which is described by second order analytical relations in space and time driven by an ubiquitous driving source (related to $\mathbf{A}^{j}$ ). This feature may provide an explanation for the ability of the balance fields to "travel" far, free from localized charges $(\rho)$ and currents $(\mathbf{j})$.

5. As a final statement supporting the definitions of $\mathbf{E}^{\rho}$ and $\mathbf{B}^{j}$ proposed herein (and the splitting that these definitions induce in the total electromagnetic fields $\mathbf{E}^{T O T}$ and $\mathbf{B}^{T O T}$ ), those still favoring the traditional option of retarded potentials are hereby challenged to derive the local (differential, or microscopic) Maxwell-Gauss and MaxwellAmpère field equations for electric and magnetic fields emerging from the retarded potentials:

\footnotetext{
${ }^{34}$ The extension is arbitrary in the sense that - contrary to the application of the Lorentz gauge to $\phi^{\rho}$ and $\mathbf{A}^{j}$, which fully satisfies the equation of continuity of electric chargesthere appears to be no argument of a physical nature justifying its application to $\Delta \phi$ and $\Delta \mathbf{A}$.
} 


$$
\phi(t, \mathbf{x})=\frac{1}{4 \pi \varepsilon_{0}} \int_{V^{\prime}} \frac{\rho\left(t_{r e t}^{\prime}, \mathbf{x}^{\prime}\right)}{\left|\mathbf{x}-\mathbf{x}^{\prime}\right|} d V^{\prime}
$$

and

$$
\mathbf{A}(t, \mathbf{x})=\frac{\mu_{0}}{4 \pi} \int_{V^{\prime}} \frac{\mathbf{j}\left(t_{r e t}^{\prime}, \mathbf{x}^{\prime}\right)}{\left|\mathbf{x}-\mathbf{x}^{\prime}\right|} d V^{\prime}
$$

where

$$
t_{\text {ret }}^{\prime}=t-\frac{\left|\mathbf{x}-\mathbf{x}^{\prime}\right|}{c}
$$

instead of deriving them for fields that emerge from the potentials defined by equations (3.8) and (3.33) respectively.

6. The exact point where Maxwell's error lies, is in his declaration:

"This equation is true only if we take $u, v$ and $w$ as the components of that electric flow which is due to the variation of electric displacement as well as true conduction."

Adding the time-derivative of the electric displacement is not the only way, but just one of the many possible ways, to make a conduction current solenoidal. By restricting his analysis to this solution -and this solution only- Maxwell assumed $\lambda_{1}=\lambda_{2}=1$ and closed unforeseen doors. 


\section{REFERENCES}

1. Sainz, Norberto. "Inertial Relativity - A Functional Analysis Review". Proyecciones (Antofagasta), ago. 2005, Vol. 24, No.2, pp. 121-152. ISSN 0716-0917.

2. Einstein, Albert. "Zur Elektrodynamik Bewegter Körper", §3. Spanish translation by Fidel Alsina F. and Damián Canales F. of original paper published in Annalen der Physik 17, 891 (1905). "La Relatividad", Emecé Editores, B. Aires, (1950).

3. Ibid. [6], $\S 6$.

4. Einstein, Albert. "Die Grundlage Der Allgemeinen Relativitätstheorie", §2-4. Spanish translation by Fidel Alsina F. and Damián Canales F. of original paper published in Annalen der Physik 49, 769 (1916). "La Relatividad", Emecé Editores, B. Aires, (1950).

5. Maxwell, James. "A Dynamical Theory of the Electromagnetic Field". Edition by T. Torrance of original paper published in Philosophical Transactions of the Royal Society of London 155, 459-512 (1865). Wipf \& Stock Publications, Eugene, OR, (1996).

6. Hertz, Heinrich. "Untersuchungen über die Ausbreitung der Elektrischen Kraft", English translation by D.E. Jones of Hertz' 1982 collection of publications in Wiedemann's Annalen. "Electric Waves", Macmillan and Co., London, (1893).

7. Ibid. [5] (3.34) pp 49.

8. Maxwell, James. "A Treatise on Electricity and Magnetism". Oxford, at the Clarendon Press, (1873).

9. Ampère, A.-M. "Sur la théorie mathématique des phénomènes électrodynamiques uniquement déduite de l'expérience", Mém. Acad. R. Sci. Inst. France, ser.2, 6, pp. 175-388, (1827).

10. Neumann, F.E. "Allgemeine Gesetze der inducirten elektriche Ströme", Abh. K. Akad. Wiss. Berlin, pp. 1-87, (1845).

11. Neumann, F.E. "Über ein allgemeines Princip der mathematischen Theorie inducirter electricher Ströme", Abh. K. Akad. Wiss. Berlin, pp. 1-72, (1847). 
12. Weber, W. "I. Elektrodynamische Maassbestimmungen", Ann. Phys. Chem. 73, pp. 193-240, 1848. (Shortened version of the 1846 paper published in Abh. K. Sächsischen Gesellschaft Wiss., Leipzig 1846).

13. Weber, W. Elektrodynamische Maassbestimmungen (Weidmann, Leipzig). (A collection of seven papers, with the same main title but different subtitles, dating from 1846 to 1878. The first part, pp. 1-170, is from Abh. K. Sächsischen Gesellschaft Wiss., Leipzig, 1846).

14. Helmholtz, H. "Ueber die Bewegungsgleichungen der Elektricität für ruhende leitende Körper". J. Reine Angew. Math. 72, pp. 57-129, (1870).

15. Faddeev, L. D., Popov, V. N. "Feynman diagrams for the Yang-Mills fields", Phys. Lett. B 25, pp. 29-30, (1967).

16. Schoenmaker, W., Magnus, W., Meuris, P., "Ghost fields in classical gauge theories", Phys. Rev. Lett. 88 (18), (2002).

17. Lorentz, H. A. "La théorie électromagnétique de Maxwell et son application aux corps movants". Arch. Néerl. Sc. 25, pp. 363-552, (1892).

18. Ibid. [8]; pp 231.

19. Ibid. [8]; pp 232 .

20. Ibid. [8]; pp 229.

21. Boudenot, J.-C. "Ampère au coeur de la physique du début du XIXème siècle". Repère: L'histoire de l'électricité. REE Revue de l'Électricité et de l'Électronique $\mathrm{N}^{o}$ 05, mai, (2003).

22. Thidé, Bo. "Electromagnetic Field Theory", pp 6-10. Upsilon Books, Uppsala University, Sweden, (1997).

23. Fock, V. "Über die invariante Form der Wellen- und der Bewegungsgleichungen für einen geladenen Massenpunkt", Z. Phys. 39, pp. 226-232, (1926).

24. Jackson, J. D., Okun, L. B. "Historical roots of gauge invariance", Reviews of Modern Physics 73, pp. 663-680, (2001). 


\section{NORBERTO SÁINZ}

Pontificia Universidad Católica de Valparaíso

Facultad de Ingeniería

Escuela de Ingeniería Industrial

Escuela de Ingeniería Eléctrica

Avda. Brasil 2241

Valparaíso

Chile

e-mail : norberto.sainz@ucv.cl 